\section{Reply to P.F. Pinsky, C.P. Crum, and M.W. McIntosh et al}

We welcome the opportunity to respond to comments raised by colleagues in the field to our recent publication. ${ }^{1}$ We thank $\mathrm{Crum}^{2}$ for his interest and insights. He highlights that highgrade serous carcinomas may arise through two routes: one from precursor lesions in the fallopian tube, such as serous tubal intraepithelial carcinoma, and the other from as-yet undefined Mullerian sources. The site of origin would determine the extent to which opportunistic salpingectomy may protect women. We share the view of Crum that this is a key issue, given the increasing popularity of this procedure as a preventive measure. We seek to address this through a randomized controlled trial.

Crum $^{2}$ is correct that United Kingdom Collaborative Trial of Ovarian Cancer Screening (UKCTOCS) could have provided a unique perspective on the origins of ovarian cancer, because women with screen-detected ovarian/tubal/primary peritoneal cancers were diagnosed earlier in their disease evolution than were symptomatic patients in clinical case series. However, the trial was designed in 2000, before the emergence of the dualistic model of high-grade serous carcinomas. Therefore, at surgery, there was no recommendation or funding for the tubes and ovaries to be subjected to the sectioning and extensively examining of the fimbriated end (SEE-FIM) protocol, which was formally described in $2006 .{ }^{3}$ We are in the process of collating tissue blocks from women diagnosed with ovarian/tubal/primary peritoneal cancer during the course of the trial for further research. At this stage, we are unable to estimate what proportion of the tumors might be available for detailed pathologic examination of the fallopian tube; however, we, like Crum, remain hopeful that there will be an eligible subset.

We would like to raise one point of clarification. $\mathrm{Crum}^{2}$ states that follow-up studies will be required to establish whether the outcome of multimodal screening will improve survival. The primary outcome measure of UKCTOCS is disease-specific mortality, and we will be reporting this at the end of the year.

The concerns of Pinsky ${ }^{4}$ are related to our definition of specificity. He suggests that specificity should be based on the results of the primary screening. We have a major difference of opinion in this regard. We share the United Kingdom National Population Screening Programme principle that screening is a process $^{5}$ that consists of multiple steps and not just of the primary screen. For population screening to be effective, the entire process needs to be centrally managed with a strict, time-dependent protocol and quality assurance. In keeping with this, all participants in UKCTOCS consented to the whole process and not to a single test. We, therefore, have reported the performance characteristics for multimodal screening in its entirety.

Although we agree with Pinsky ${ }^{4}$ that it is important to describe the proportion of women who would need repeat testing, we do not agree this should be the primary measure of specificity. At the start of the trial, we calibrated the risk cutoffs after the primary screening so that $10 \%$ to $15 \%$ of women would be at intermediate risk of ovarian cancer and would have a repeat blood test, and $2 \%$ of women would be at elevated risk of ovarian cancer and would go on to have a transvaginal scan and repeat blood test. This was described in the patient information sheet and was known to volunteers before they consented to participate.

Pinsky ${ }^{4}$ is correct that any screening result that requires follow-up, including a repeat screen, carries potential harms and costs and should be counted. Thus, an independent psychosocial study has been undertaken alongside UKCTOCS to assess potential harm at each stage. Findings suggest that screening did not raise anxiety and that repeat tests after the annual screening, overall number of repeat tests, and more intensive screening (eg, transvaginal scan and blood test $v$ repeat blood test) did not significantly affect anxiety compared with routine annual screening. Surgical procedures that, in our trial, defined performance characteristics (both sensitivity and specificity) did, however, cause changes in anxiety. ${ }^{6}$ In addition, a separate health economic analysis will report the cost effectiveness of the entire screening process, including all repeat tests and not just the annual screening. The economic analysis will be available after the publication of the mortality results.

The first issue that McIntosh et $\mathrm{al}^{7}$ raise relates to our title and core message, which they suggest are misleading. We would like to clarify that these reflect the actual results of the largest-ever prospective trial of multimodal ovarian cancer screening, which involved 296,911 annual incidence screenings in 46,237 women. We strongly believe that the comparison of our outcomes to what has been previously achieved with ovarian screening in the Prostate, Lung, Colorectal, and Ovarian Cancer Screening Trial, ${ }^{8}$ for which a single-threshold (ST) rule was applied, was essential to assess whether any progress had been made. Ultimately, the effectiveness of screening depends on its impact on mortality, and our group has led the effort to deliver a definitive answer. The mortality impact publication should be available by the end of this year.

Although some of the group have an association with Abcodia, which is commercializing the Risk of Ovarian Cancer Algorithm (ROCA), the company was not in any way involved in the funding or conduct of UKCTOCS and had no influence on the analysis and interpretation of our data.

McIntosh et $\mathrm{al}^{7}$ state that sensitivity and specificity were not calculated in a compatible fashion. As mentioned in the response to remarks by Pinsky, screening is a process, and we clearly stated the sensitivity $(85.8 \%)$ and specificity $(99.8 \%)$ of the multimodal approach for detection of invasive epithelial ovarian cancer (iEOC) in the abstract. We then went on to compare the ST rule and ROCA on the primary screen, and we stated that ROCA detected $87.1 \%$ of the iEOC and that the ST rule would have identified $41.3 \%$ to $66.5 \%$, depending on the cutoff value. No specificity was stated for either. In the Results section and in Figure 4 of our article, ${ }^{1}$ sensitivity and specificity for ROCA and the ST rule at multiple cutoffs are clearly provided: sensitivity of ROCA at annual screening was $87.1 \%$ (95\% CI, $80.8 \%$ to $91.9 \%$ ), and specificity was $87.6 \%$, and those of the ST rule with an annual CA- 125 cutoff of $20.99 \mathrm{U} / \mathrm{mL}$ were $68.4 \%$ (95\% CI, $58.4 \%$ to $73.8 \%$ ) and $87.6 \%$, respectively. 
McIntosh et $\mathrm{al}^{7}$ correctly noted that women should not interpret ROCA sensitivity or detection rates to mean that $85.8 \%$ of screening-detected iEOCs are treated before symptoms arise. As is well known in the context of screening, symptoms are reported by a proportion of apparently well individuals who present for screening. This is likely to be magnified in the context of ovarian cancer, for which the symptoms are ill defined despite significant efforts. We have already undertaken a detailed analysis of the presence of symptoms in women with screening-detected ovarian/tubal/peritoneal cancers in UKCTOCS, and this analysis has been accepted for presentation at the European Society of Gynaecological Oncology (ESGO) annual meeting in October 2015.

We regret the attempt by McIntosh et $\mathrm{al}^{7}$ to extract and analyze incomplete data from our published figure. This does not align with the academic spirit in which we and others offer data for publication in scientific journals. UKCTOCS is ongoing (follow-up until 2024), and the trial team is currently undertaking numerous analyses of the data. In due course, we hope to collaborate with interested researchers. Furthermore, the presented analysis is flawed, because the authors have no access to the ROCA risk classification of previous annual screens. McIntosh et $\mathrm{al}^{7}$ assumed that the ROCA-derived risk was normal on previous screens. This is incorrect. A blinded comparison of published and new serial algorithms with the entire UKCTOCS multimodal data set was planned in 2011 as part of our ongoing research efforts to improve ovarian cancer screening strategies and is almost complete. A publication with an accurate comparison of different strategies should be freely available early next year.

We agree with McIntosh et $\mathrm{al}^{7}$ on the need to develop strategies to manage elevated risk on the basis of serial marker levels and negative imaging. This has been a core issue that we have tackled and discussed widely in international meetings since the start of UKCTOCS in 2001. In the trial, we recommended surgery if the risk was 1 or greater in 5 despite normal cross-sectional imaging. The authors suggest that new molecular imaging techniques are the only way forward. Although these techniques are an avenue worth exploring, we are of the opinion that much can also be gained by the exploration of serial change in other markers alongside CA-125. This is being systematically investigated in a blinded case-control study that uses all of the serial samples from women in the multimodal group who have developed ovarian/ tubal/peritoneal cancers in the course of UKCTOCS.

In conclusion, UKCTOCS definitively demonstrates that multimodal screening with ROCA detects ovarian cancer with high sensitivity and specificity. Later this year, we will know whether this translates into a reduction in ovarian cancer mortality.

Usha Menon, Jatinderpal K. Kalsi, Aleksandra Gentry-Maharaj, Andy Ryan, Matthew Burnell, and Max Parmar
University College London, London, United Kingdom

\section{Lesley Fallowfield}

University of Sussex, Falmer, United Kingdom

\section{Stuart Campbell}

Create Health Clinic, London, United Kingdom

\section{Alistair McGuire}

London School of Economics, London, United Kingdom

\section{Steven Skates}

Massachusetts General Hospital and Harvard Medical School, Boston, MA

\section{Ian Jacobs}

University College London, London, United Kingdom; and University of New South Wales, Sydney, New South Wales, Australia

\section{ACKNOWLEDGMENT}

Core funding provided by Medical Research Council, Cancer Research UK, Department of Health, with additional support from the National Institute for Health Research, University College London, Hospital Biomedical Research Centre, and the Eve Appeal. Written on behalf of United Kingdom Collaborative Trial of Ovarian Cancer Screening (UKCTOCS) trialists.

\section{AUTHORS' DISCLOSURES OF POTENTIAL CONFLICTS OF INTEREST}

Disclosures provided by the authors are available with this article at www.jco.org.

\section{REFERENCES}

1. Menon U, Ryan $A$, Kalsi J, et al: Risk algorithm using serial biomarker measurements doubles the number of screen-detected cancers compared with a single-threshold rule in the United Kingdom Collaborative Trial of Ovarian Cancer Screening. J Clin Oncol 33:2062-2071, 2015

2. Crum CP: Preventing ovarian cancer. J Clin Oncol 34:198-199, 2016

3. Lee $Y$, Medeiros F, Kindelberger D, et al: Advances in the recognition of tubal intraepithelial carcinoma: Applications to cancer screening and the pathogenesis of ovarian cancer. Adv Anat Pathol 13:1-7, 2006

4. Pinsky PF: Definition of specificity in a screening trial. J Clin Oncol 34:198, 2016

5. Public Health England: NHS population screening explained, 2013. www gov.uk/guidance/nhs-population-screening-explained

6. Barrett J, Jenkins V, Farewell V, et al: Psychological morbidity associated with ovarian cancer screening: Results from more than 23,000 women in the randomized trial of ovarian cancer screening (UKCTOCS). BJOG 121:1071-1079, 2014

7. Mclntosh MW, Drescher C, Fitzgibbon MM: Ovarian cancer early detection needs better imaging, not better algorithms or biomarkers. J Clin Oncol 34: 199-200, 2016

8. Buys SS, Partridge $E$, Black $A$, et al: Effect of screening on ovarian cancer mortality: The Prostate, Lung, Colorectal and Ovarian (PLCO) Cancer Screening randomized controlled trial. JAMA 305:2295-2303, 2011

DOI: 10.1200/JCO.2015.64.1365; published online ahead of print at www.jco.org on November 16, 2015 


\section{AUTHORS' DISCLOSURES OF POTENTIAL CONFLICTS OF INTEREST}

Reply to P.F. Pinsky, C.P. Crum, and M.W. McIntosh et al

The following represents disclosure information provided by authors of this manuscript. All relationships are considered compensated. Relationships are self-held unless noted. I = Immediate Family Member, Inst = My Institution. Relationships may not relate to the subject matter of this manuscript. For more information about ASCO's conflict of interest policy, please refer to www.asco.org/rwc or jco.ascopubs.org/site/ifc.

\section{Usha Menon}

Stock or Other Ownership: Abcodia

Research Funding: Abcodia (Inst)

Patents, Royalties, Other Intellectual Property: European Patent No. EP10178345.4

\section{Jatinderpal K. Kalsi}

No relationship to disclose

Aleksandra Gentry-Maharaj

No relationship to disclose

Andy Ryan

No relationship to disclose

Matthew Burnell

No relationship to disclose

\section{Max Parmar}

Research Funding: Astellas Pharma (Inst), AstraZeneca (Inst), Bayer AG (Inst), Baxter (Inst), Janssen Pharmaceuticals (Inst), Novartis (Inst), Roche (Inst), Sanofi (Inst)

\section{Lesley Fallowfield}

Honoraria: Amgen, Roche UK, Roche, Bayer AG, TEVA Pharmaceutical Industries

Consulting or Advisory Role: Amgen, Sanofi

Speakers' Bureau: Amgen, Roche UK, Roche, Astellas Pharma Research Funding: Roche (Inst), Boehringer Ingelheim (Inst), GlaxoSmithKline (Inst), Roche UK (Inst), Sanofi (Inst)

Travel, Accommodations, Expenses: Amgen, Roche UK, Roche

\section{Stuart Campbell}

No relationship to disclose

\section{Alistair McGuire}

No relationship to disclose

\section{Steven Skates}

Stock or Other Ownership: SISCAPA Assay Technologies

Consulting or Advisory Role: Abcodia

Speakers' Bureau: AstraZeneca

Patents, Royalties, Other Intellectual Property: Abcodia (Inst)

Ian Jacobs

Employment: Women's Health Specialists

Leadership: Abcodia

Stock or Other Ownership: Abcodia

Consulting or Advisory Role: Abcodia

Patents, Royalties, Other Intellectual Property: ROC algorithm (Inst) 